\title{
AAV-mediated expression of NFAT decoy oligonucleotides protects from cardiac hypertrophy and heart failure
}

\author{
Anca Remes ${ }^{1,3,4}$. Andreas H. Wagner ${ }^{3}$. Nesrin Schmiedel ${ }^{1,4} \cdot$ Markus Heckmann $^{2}$ - Theresa Ruf ${ }^{1,2} \cdot$ Lin Ding $^{1,4}$. \\ Andreas Jungmann $^{2} \cdot$ Frauke Senger $^{1,4} \cdot$ Hugo A. Katus $^{2} \cdot$ Nina D. Ullrich $^{3} \cdot$ Norbert Frey $^{1,2,4} \cdot$ Markus Hecker $^{3}$. \\ Oliver J. Müller ${ }^{1,4}$ (1)
}

Received: 4 August 2020 / Accepted: 18 May 2021 / Published online: 4 June 2021

(c) The Author(s) 2021

\begin{abstract}
Previous studies have underlined the substantial role of nuclear factor of activated T cells (NFAT) in hypertension-induced myocardial hypertrophy ultimately leading to heart failure. Here, we aimed at neutralizing four members of the NFAT family of transcription factors as a therapeutic strategy for myocardial hypertrophy transiting to heart failure through AAV-mediated cardiac expression of a RNA-based decoy oligonucleotide (dON) targeting NFATc1-c4. AAV-mediated dON expression markedly decreased endothelin-1 induced cardiomyocyte hypertrophy in vitro and resulted in efficient expression of these dONs in the heart of adult mice as evidenced by fluorescent in situ hybridization. Cardiomyocyte-specific dON expression both before and after induction of transverse aortic constriction protected mice from development of cardiac hypertrophy, cardiac remodeling, and heart failure. Singular systemic administration of AAVs enabling a cell-specific expression of dONs for selective neutralization of a given transcription factor may thus represent a novel and powerful therapeutic approach.
\end{abstract}

Keywords Heart failure $\cdot$ Cardiac hypertrophy $\cdot$ Transcription factor $\cdot$ NFAT $\cdot$ Decoy oligonucleotide $\cdot$ Adeno-associated virus

\section{Introduction}

Although treatment options have significantly improved prognosis and quality of life of patients diagnosed with heart failure, current therapies at best offer reduced disease progression but not a real cure [30]. Cardiac hypertrophy is initiated by various stress signals, such as growth factors

Anca Remes, Andreas H. Wagner, Markus Hecker and Oliver J. Müller contributed equally to this work.

Oliver J. Müller

oliver.mueller@uksh.de

1 Department of Internal Medicine III, University Hospital Schleswig-Holstein and University of Kiel , Arnold-Heller-Str. 3 , Kiel, Germany

2 Internal Medicine III, University Hospital Heidelberg, Heidelberg, Germany

3 Institute of Physiology and Pathophysiology, Heidelberg University, Heidelberg, Germany

4 German Centre for Cardiovascular Research, Partner Site Hamburg/Kiel/Lübeck , Kiel, Germany and pressure or volume overload [12]. Although this state is compensatory by temporarily preserving cardiac output, sustained pathological myocardial hypertrophy is associated with increased risk of heart failure, arrhythmias and sudden death [12, 42].

Myocardial hypertrophic growth is a highly intricate process governed by activation of the calcineurin-NFAT signaling pathway [32, 45]. Following dephosphorylation by calcineurin, transcriptional activation of the NFAT family members $\mathrm{c} 1$ to $\mathrm{c} 4$ regulates central processes leading to heart failure, such as myocyte growth, extracellular matrix deposition and re-activation of the fetal gene program [17, 41]. A number of studies have delineated the major importance of NFAT in the development of cardiac hypertrophy. Cardiac-specific activation of the calcineurin-NFAT pathway was sufficient to induce myocardial hypertrophy [33], whereas both pharmacological inhibition [24] and genetic deletion of NFATc2 and c3 [2, 46] alleviated pathological remodeling in animal models. Therefore, reducing the transcriptional activity of NFAT could represent a valuable treatment option for cardiac hypertrophy and heart failure. 
One approach of interfering with abnormal activity of transcription factors for therapeutic purposes is represented by decoy oligodeoxynucleotides (dODNs). These are short (10-25 bp) sequences of double-stranded DNA mimicking the consensus binding site of the target transcription factor, which upon cellular entry specifically neutralize it and hence inhibit transcription of its target genes [15]. These nucleic acid-based drugs have been proven to be safe and effective in various preclinical and clinical trials focusing on diverse disease models [29]. Until now, the most frequent route of administration employed is local delivery, which requires sustained application of the active dODNs [8, 9]. In terms of future therapy and depending on the target organ, this approach is not always feasible.

Here, we show that single injection of an adeno-associated viral vector (AAV9) is sufficient to express a decoy hairpin RNA oligonucleotide ( $\mathrm{dON})$ neutralizing NFATc1c4 in cardiomyocytes in vivo. This singular treatment before or after subjecting mice to transverse aortic constriction (TAC) was associated with a dramatic improvement in cardiac function and reduced remodeling, and therefore broaden the paradigm of decoy oligo(deoxy)nucleotides as nucleic acid-based therapeutics.

\section{Materials and methods}

\section{Primary cardiomyocytes isolation and culture}

Primary neonatal rat cardiomyocytes (NRVCMs) were isolated according to previously established protocols [22]. Experiments were executed under the guidelines from Directive 2010/63/EU of the European Parliament on the protection of animals used for scientific purposes with approval of the local authorities in Kiel (permission number 1085). In brief, left ventricles from 2-day-old Wistar rats were harvested and digested in the presence of $0.6 \mathrm{mg} / \mathrm{mL}$ pancreatin (Sigma-Aldrich, Munich, Germany) and $0.5 \mathrm{mg} /$ $\mathrm{mL}$ collagenase II (Worthington Biochemical Corporation, Lakewood, USA) at $37{ }^{\circ} \mathrm{C}$. The resulting cell suspension was filtered through a cell strainer and afterwards centrifugation on a Percoll gradient (GE Healthcare, Chicago, USA) was performed to ensure separation from fibroblasts. Next, NRVCMs were cultured in complete DMEM medium containing $10 \%$ fetal bovine serum, $2 \mathrm{mmol} / \mathrm{L}$ L-glutamine, 100 $\mathrm{U} / \mathrm{mL}$ penicillin, and $100 \mu \mathrm{g} / \mathrm{mL}$ streptomycin (Thermo Fischer Scientific, Darmstadt, Germany).

\section{DNA dODN technology}

DNA-based dODNs were designed to form a hairpin structure by intramolecular hydrogen bond formation and to contain the promoter binding site of NFAT1-4 (Biomers,
Ulm, Germany). A 5'-Atto-590 labeled dODN was used for assessment of cellular uptake efficiency. The sequences of the dODNs used in our study are as follows: hpNFAT cons dODN: 5'-GAGTGGAAACATACAGCCACTGAA ACAGTGGCTGTATGTTTCCACTC-3' and hpNFAT mut dODN: 5'-GAGCTTAAACATACAGCCACTGAAACA GTGGCTGTATGTTTCCACTC-3'. Decoys were dissolved in sterile TEN buffer to a concentration of $500 \mu \mathrm{mol} / \mathrm{L}$, incubated at $95{ }^{\circ} \mathrm{C}$ for $5 \mathrm{~min}$, followed by gradual cooling down, to induce hybridization of complementary base pairs and hairpin structure formation. Successful hybridization was proven by agarose gel electrophoresis.

\section{AAV production}

Sequences of hpNFAT cons and mut dONs were synthesized (GeneArt, Thermo Fischer Scientific, Darmstadt, Germany) and cloned between XhoI and SalI restriction sites of the dON-generating vector, as previous described [27]. The gene synthesis sequences are presented below: hpNFATcons dON: 5'-AGGCGCCCTGCAATATTTGCATGT CGCTATGTGTTCTGGGAAATCACCATAAACGTGAAA TGTCTTTGGATTTGGGAATCTTATAAGTTCTGTATG AGACCACAGTCGACGAGTGGAAACATACAGCCA CTGAAACAGTGGCTGTATGTTTCCACTCCACCGCAG TTTCGACCTCGAGA-3'; hpNFATmut dON: 5'-AGGCGC CCTGCAATATTTGCATGTCGCTATGTGTTCTGGGAA ATCACCATAAACGTGAAATGTCTTTGGATTTGGGAA TCTTATAAGTTCTGTATGAGACCACAGTCGACGAGC TTAAACATACAGCCACTGAAACAGTGGCTGTATGTT TCCACTCCACCGCAGTTTCGACCTCGAGA-3'. AAV6 and AAV9 vectors were produced and purified as described elsewhere [16].

\section{Decoy ODN and AAV treatment}

Decoy ODNs were added in serum-free cell culture medium to a concentration of $10 \mu \mathrm{mol} / \mathrm{L}$ prior to ET-1 stimulation. AAV transduction was conducted at a M.O.I. of $10^{5} \mathrm{vg} / \mathrm{cell}$.

\section{Fluorescent in situ hybridization}

The generation of hpNFAT RNA dONs following AAV9 transduction was verified in $5 \mu \mathrm{m}$ myocardial frozen sections by fluorescent in situ hybridization according to standard protocols [47]. A molecular beacon with complementary sequence to the dON was used as a probe (Biomers, Ulm, Germany).

\section{Quantitative real-time PCR}

Total RNA was extracted from adherent cells or tissue using RNeasy Mini Kit (Qiagen, Hilden, Germany) following the 
manufacturer's instructions. First-strand synthesis of cDNA was completed using Omniscript Reverse Transcriptase kit (Qiagen, Hilden, Germany) and OligodT primers (Promega, Mannheim, Germany), starting from equal amount of RNA for each sample. Left ventricular tissues were processed using QIAschredder (Qiagen, Hilden, Germany). SYBR Green (Qiagen, Hilden, Germany) qRT-PCR was performed using Qiagen Rotor-Gene machine. The sequences of primers used in this study and corresponding annealing temperatures are presented in Table1. RPL32 was used as a housekeeping gene for normalization of gene expression.

\section{Histological analyses}

Hearts were fixed in $4 \% p$-formaldehyde (PFA) overnight at $4{ }^{\circ} \mathrm{C}$ and embedded in paraffin prior to histological processing. For visualization of collagen fibers, sections were subjected to Masson's Trichrome Staining (Sigma Aldrich, Munich, Germany). All sections were stained at the same time to avoid variation in staining intensity. Images were taken in random areas of the left ventricle using a brightfield microscope (Leica DM500, Leica Microsystems, Mannheim, Germany). Percentage blue area out of total section dimension was measured by ImageJ (1.51p, National Institute of Health, US) and further used for analysis.

\section{Cell size measurement}

In vitro NRVCM area measurement was performed according to established procedures. Immunofluorescent staining of $\alpha$-actinin (Sigma-Aldrich, Munich, Germany) was performed in cell preparations according to standard protocols and images were taken with $10 \times$ magnification (BZ-9000 Keyence, Itasca, USA). Cardiomyocytes were identified to be positive for the stained marker, while fibroblasts, occasionally present in these primary cell cultures, were negative. A total of 200 cells were analyzed in each treatment group. Cell size was measured using BZ-II Analyzer (version 2.1, HybridCellCount module).

To determine cardiomyocyte cross section in the myocardium, frozen sections were subjected to cell membrane staining with fluorescently labeled WGA (Thermo Fischer Scientific, Bremen, Germany). Images were taken using a confocal microscope (Zeiss LSM 800, Oberkochen, Germany) and relative cell area was analyzed by ImageJ.

\section{Transverse aortic constriction}

Animal experiments were carried out under the guidelines from Directive 2010/63/EU of the European Parliament on the protection of animals used for scientific purposes with approval of the regional authorities in Karlsruhe (G180/12) and Kiel (V312-7224.121-4). Animals were kept under standard conditions (Interfaculty Biomedical Facility, Heidelberg, Germany and the animal facility of the University Medical Center Schleswig-Holstein, Kiel) with 12-h light, 12-h night cycle; water and food was offered ad libitum. Transverse aortic constriction (TAC) was performed in 10 weeks old C57BL/6 N mice as previously described [26], with a 27-gage needle for stenosis induction. Successful ligation was confirmed by measuring the right carotid/ left carotid flow velocity ratio. Echocardiography was performed at baseline, 2, 4, and 6 weeks (prophylaxis study) or at 2 and 6 weeks (therapy study) post TAC using a VisualSonics Vevo 2100 imaging system and the $40 \mathrm{~Hz}$ MS550D micro scan transducer. The measurements and data analyses were performed by an experimenter blinded to the treatment. Long axis and M-mode short axis cine loops were recorded. Left ventricular ejection fraction $(\mathrm{EF})$ and left ventricular posterior wall diameter at diastole (LVPW,d) were
Table 1 List of primers used in the study

\begin{tabular}{lll}
\hline Gene & Sequence & $\begin{array}{c}\text { Annealing } \\
\text { temperature } \\
\left({ }^{\circ} \mathrm{C}\right)\end{array}$ \\
\hline ANP & Qiagen (QT00250922) & 55 \\
BNP & Qiagen (QT00107541) & 55 \\
Col.3 & Forward: 5'-TGGTCCTCAGGGTGTAAAGG-3' & \\
EGFP & Reverse: 5'-GTCCAGCATCACCTTTTGGT-3' & \\
& Forward: 5'-AGTCCGCCCTGAGCAAAGA-3' & 60 \\
HSP70 & Reverse: 5'-TCCAGCAGGACCATGTGATC-3' & 57 \\
RCAN1 & Forward: 5'-CCCGGTGTGGTCTAGAAAACA-3' & \\
RPL32 & Reverse: 5'-CCATGAAGAAGACTTTAAATAACCTTGAC-3' & 55 \\
SMIT & Qiagen (QT01053430) & 55 \\
TGF- $\beta$ & Forward: 5'-GGGAGCAACAAGAAAACCAA-3' & 55 \\
\hline
\end{tabular}


determined using VisualSonics software. Animals were sacrificed 6 weeks after surgery. Heart weight/tibia length ratio was measured as a marker of cardiac hypertrophy, as well as lung weight/tibia length ratio for monitoring heart failure induced lung edema. Body weights were recorded daily.

\section{NFAT activity assay}

NFAT activity was analyzed using a TransAM® NFATc1 kit (Active Motif, Carlsbad, USA) according to the instructions provided by the manufacturer. A total of $15 \mu \mathrm{g}$ nuclear extract was added to each well.

\section{Immunocytochemistry}

Immunocytochemistry was used to detect NFATc1 translocation under pro-hypertrophic conditions. In brief, NRVCMs were fixed with 5\% PFA for $5 \mathrm{~min}$ and incubated for $1 \mathrm{~h}$ in a buffer containing $3.5 \%$ BSA and $0.1 \%$ Triton X-100 to block non-specific binding of antibodies. Afterwards, cells were treated with primary antibody (Santa Cruz, Heidelberg, Germany) overnight at $4{ }^{\circ} \mathrm{C}$ in a humidified atmosphere. The corresponding fluorescent dye-labeled secondary antibody (Thermo Fischer Scientific, Darmstadt, Germany) was added after a series of washing steps and incubated for $1 \mathrm{~h}$ at ambient temperature. Images were quantified using ImageJ.

\section{Western blot analysis}

Western blot analysis was conducted according to standard protocols. In brief, $20 \mu \mathrm{g}$ protein was separated by SDSPAGE and next transferred onto nitrocellulose membranes. Specific antibodies against tubulin (Sigma-Aldrich, T5168, dilution 1:10 000 in milk) and histone H3 (Cell Signaling, 4499, dilution 1:5000 in milk) were incubated overnight at $4{ }^{\circ} \mathrm{C}$. Next, membranes were treated with horseradish peroxidase-coupled secondary antibodies (Dianova, dilution 1:10 000 in milk) for $1 \mathrm{~h}$ at ambient temperature. Detection was performed using ECL Detecton reagent (Thermo Fischer Scentific).

\section{Enzyme-linked immunosorbent assay}

Enzyme-linked Immunosorbent Assay (ELISA) was performed according to the manufacturer's instruction (SigmaAldrich, Munich, Germany), starting from $50 \mu \mathrm{L}$ of plasma. Samples were diluted 1:1 with assay diluent prior to analysis.

\section{Statistical data analysis}

Statistical data evaluation and generation of graphs were made using GraphPad Prism 7 software (San Diego, California, USA). Differences between 3 or more groups were assessed using One-way ANOVA, followed by a Tukey's multiple comparisons test for particular pairs of groups. Mann-Whitney $U$ test was used to compare two groups. All data were normalized to the respective control group. A $p$ value $<0.05$ was considered significant. Data are presented as means $\pm \mathrm{SD}$ of individual experiments.

\section{Results}

\section{Hairpin NFAT decoy oligodeoxynucleotides decrease ET-1-induced cardiomyocyte hypertrophy}

First, we designed a highly specific DNA-based dODN to neutralize NFAT activity, demonstrating remarkable stability in a hairpin loop conformation (Fig. 1a). This hairpin (hp) dODN is intended to neutralize all four calcineurindependent NFATs, i.e. NFATc1-c4 but not NFAT5, which is activated independently of calcineurin. Next, we confirmed that hairpin dODNs are taken up by neonatal rat ventricular cardiomyocytes (NRVCM) in vitro, and also translocate to the nucleus to some extent (Fig. 1b). To elucidate the effects of NFAT inhibition by this dODN on cardiomyocyte hypertrophy in vitro, NRVCMs were stimulated with ET-1 subsequent to pre-incubation with the consensus or mutant control dODNs. Real-time qPCR analysis indicated that NFAT neutralization with the consensus but not the mutant control dODN significantly decreased expression of the fetal genes ANP and BNP (Fig. 1c, d). Exposure to ET-1 also resulted in a marked increase in NRVCM size, which was virtually abolished by prior incubation with the consensus but not the mutant control dODN (Fig. 1e, f).

\section{AAV6-mediated hpNFAT RNA decoy oligonucleotide expression blocks ET-1-induced cardiomyocyte hypertrophy}

Having demonstrated that neutralizing NFAT using hpNFAT dODN interferes with cardiomyocyte hypertrophy, we next aimed at developing this approach further into a continuous delivery system for the nucleic acid-based drug (Fig. 2a). For this purpose, we cloned the sequences of the designed consensus and mutant control hpNFAT dODNs downstream of the $\mathrm{H} 1$ promoter into an AAV plasmid backbone (AAVcons and AAVmut, $c f$. Figure $2 \mathrm{~b}$ ) and generated AAV serotype six vectors for in vitro studies. Next, we investigated nuclear translocation and binding of NFATc1, representative for NFATc1-4 as these NFAT family members share a conserved DNA-binding domain that specifies binding to the DNA core sequence (A/T)GGAAA [31]. While NFATc1 translocation to the nucleus of NRVCMs in response to ET-1 was unaffected (Fig. 2c, d), binding to its cognate cisregulatory elements in the promoters of its target genes, as 
Fig. 1 Hairpin NFAT dODNs exert anti-hypertrophic effect in NRVCMs. a Sequence of the designed NFAT dODN and physical properties, showing enhanced stability as a hairpin form. b Representative confocal microscope images showing the uptake of fluorescently labeled dODNs (magenta) by NRVCMs. Nuclei were stained with DAPI. Arrows point towards nuclear localization of dODNs. Scale bar represents $25 \mu \mathrm{m}$. c, d Statistical quantification of fetal gene products ANP and BNP on mRNA level. RPL32 was used as a housekeeping gene and values were normalized to non-stimulated cardiomyocytes as control. e Illustrative images of $\alpha$-actinin detection by immunocytochemistry (magenta) of NRVCMs of the depicted treatment groups and f Statistical quantification of relative cardiomyocyte size as a measure of pro-hypertrophic response. Scale bar represents $25 \mu \mathrm{m}$. $\left(n=4,{ }^{*} p<0.05,20\right.$ images analyzed/group) a

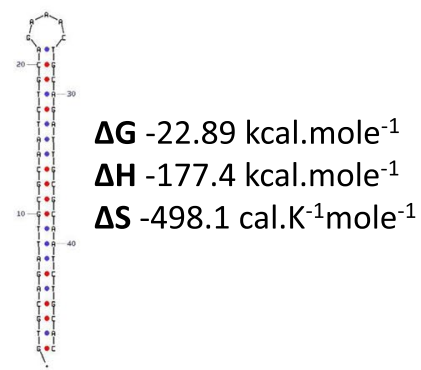

C

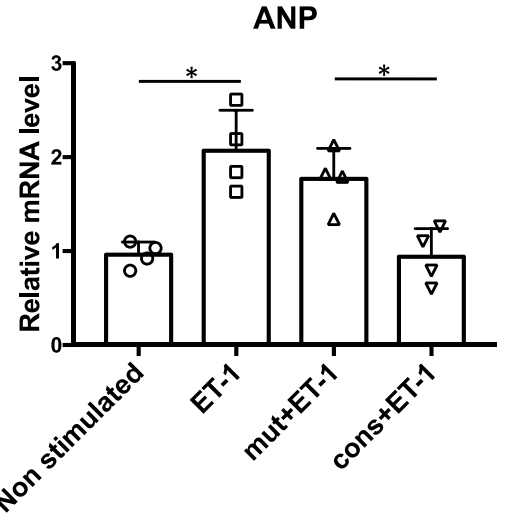

e

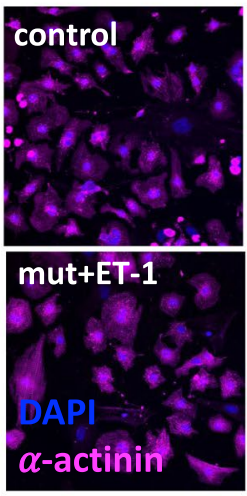

b
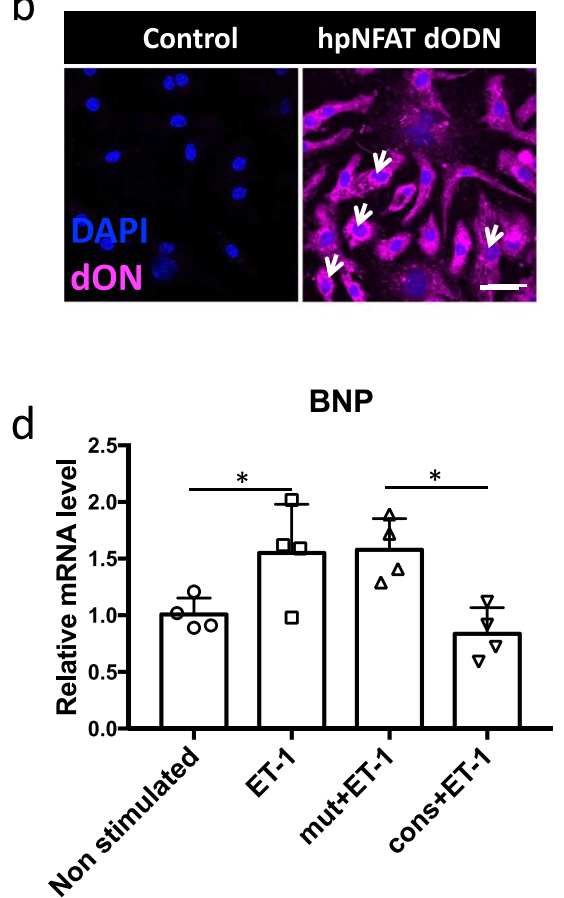

f

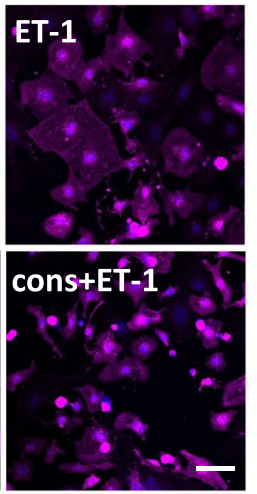

Cell size

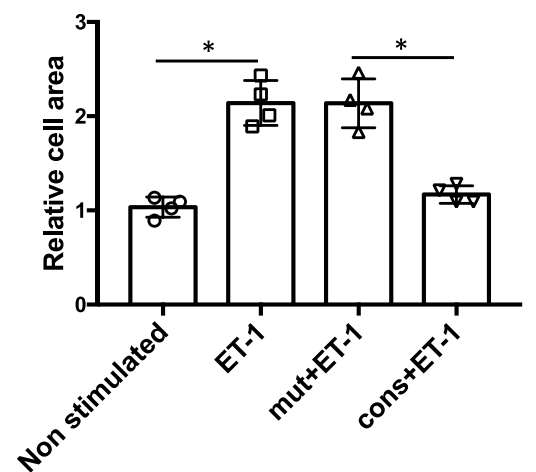

determined by an ELISA-based DNA-binding assay, was virtually abolished following AAV6cons but not AAV6mut transduction of the NRVCMs (Fig. 2e). Moreover, in AAV6cons but not AAV6mut-treated NRVCMs, ET-1-stimulated expression of the fetal genes ANP and BNP was nearly reduced to baseline (Fig. 2f, g) as was the ET-1-mediated increase in cell size (Fig. 1h, i), a parameter for the induction of hypertrophy in vitro. Collectively, these data verify that the RNA-based hpNFAT consensus dON is expressed by the cultured NRVCMs at a concentration that effectively neutralizes NFAT activity stimulated by a prototypic prohypertrophic mediator.

Specificity of the dON was further analyzed in vitro. NFAT5 plays a vital role in cardiomyocyte physiology by regulating the response to osmotic stress [1]. We therefore addressed the question whether the expressed dON neutralizes NFAT5 in cardiomyocytes and thus interferes with the expression of prototypic NFAT5 target genes. Accordingly, cardiomyocytes were exposed to $100 \mathrm{mmol} / \mathrm{L} \mathrm{LiCl}$ for $24 \mathrm{~h}$ to upregulate NFAT5-dependent gene expression. Immunofluorescence analysis (Suppl. Figure 1a) revealed that neither the DNA-based hpNFAT consensus dODN nor AAV6mediated expression of the RNA-based hpNFAT consensus dON influences NFAT5 translocation to the nucleus (Suppl. Figure 1b). Likewise, osmotic stress-induced expression of SMITS and HSP70, products of the prototypic NFAT5 target genes SLC5A3 and HSPA, was not affected by the two consensus oligonucleotides (Suppl. Figure 1c, d). 
a

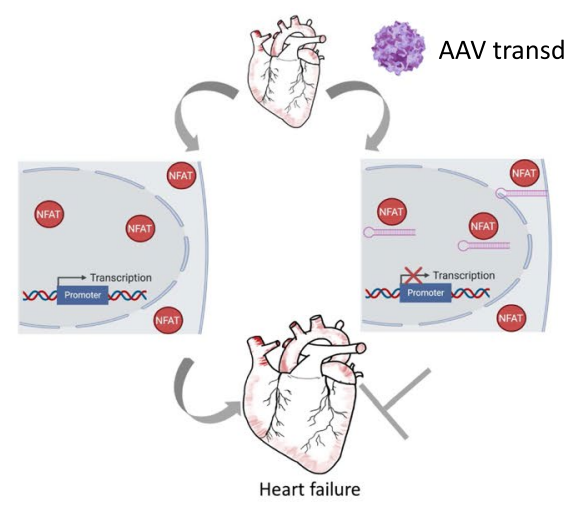

C

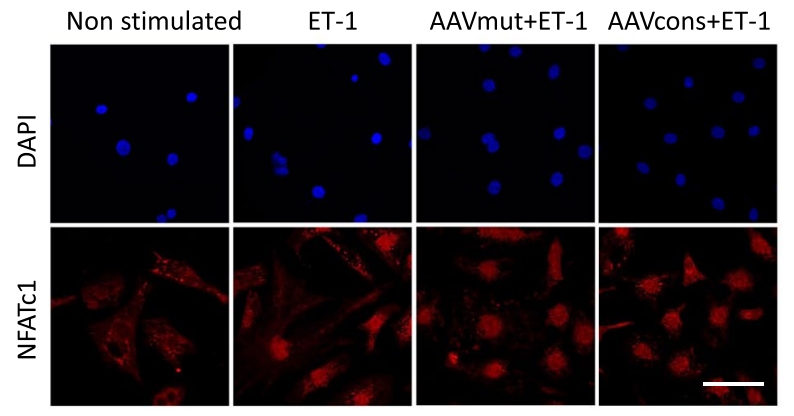

\section{f}

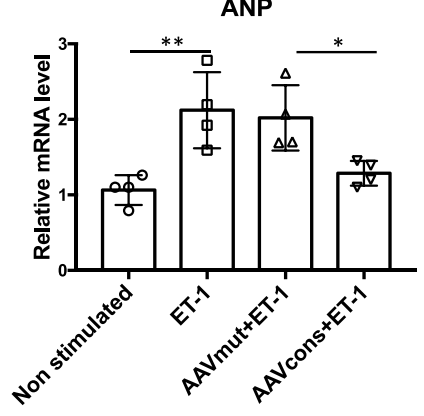

g

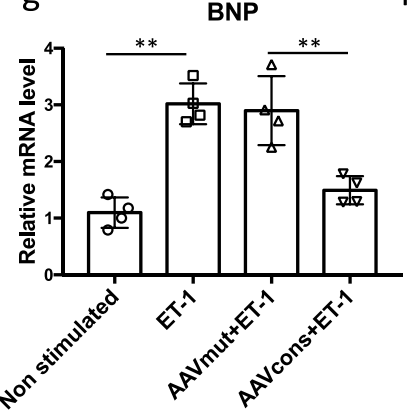

Fig. 2 AAV6-mediated hpNFAT dONs delivery in NRVCMs reduces the pro-hypertrophic response to ET-1. a Graphical representation showing the project concept. Binding of members of the NFAT transcription factor family to specific promoter regions play a central role in the hypertrophic gene expression program (left). AAV-mediated cardiac expression of RNA-based dONs aims to neutralize NFATc1-4 and prevent its interaction with NFAT binding sites in the genome (right). b Schematic representation of dON generation following AAV transduction. Decoy ONs are highly stable as hairpin structure. ON seq: sequence of the designed NFAT dON. c Representative images showing NFATc1 (red) expression in NRVCMs in depicted

\section{AAV9-mediated cardiomyocyte-specific hpNFAT RNA decoy oligonucleotide expression prevents pressure overload-induced cardiac hypertrophy and heart failure}

To validate the above results in vivo, AAV9 vectors d

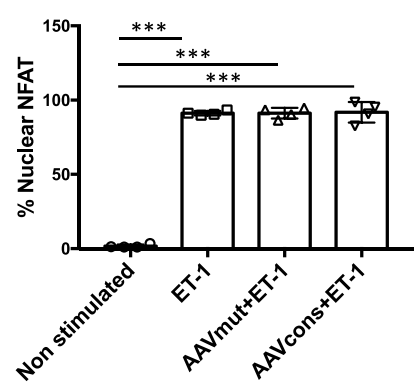

$\mathrm{h}$

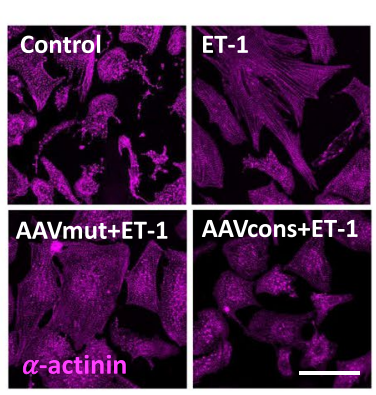

e

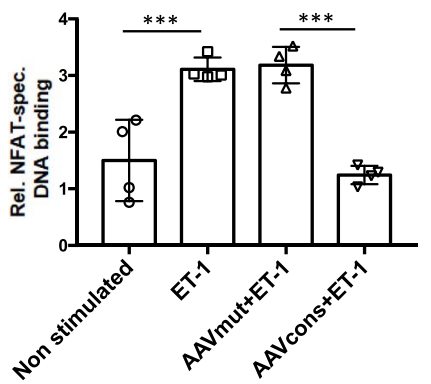

i

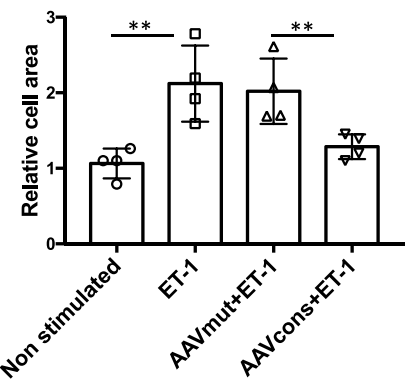

treatment groups. DAPI served as a nuclear marker. Scale bar represents $25 \mu \mathrm{m}$. d Statistical quantification of nuclear NFAT abundance following ET-1 stimulation. e Determination of NFAT-specific DNAbinding capacity in nuclear extracts of NRVCMs. $\mathbf{f}, \mathbf{g}$ Quantification of ANP and BNP expression levels following AAV6 transduction as markers of pro-hypertrophic response. $\mathbf{h}$ Representative immunocytochemistry images showing $\alpha$-actinin staining (magenta). I Statistical quantification of cardiomyocyte cross-sectional area in frozen sections stained with WGA $(n=4, * p<0.05, * * p<0.01, * * p<0.0120$ images analyzed/group)

expressing hpNFAT consensus or mutant control ON were intravenously injected in mice two weeks prior to induction of cardiac hypertrophy through TAC (Fig. 3a). This allowed expression of the RNA-based dON before induction of cardiac hypertrophy (preventive treatment approach). Successful generation of the dON was verified in an independent 
a

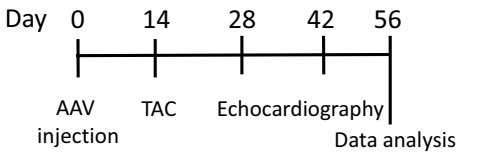

b

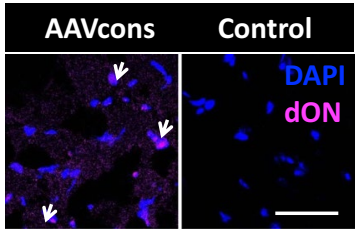

e

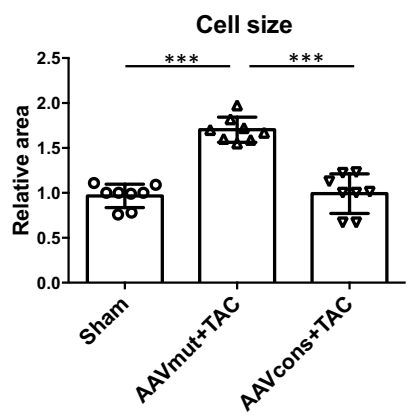

$\mathrm{h}$

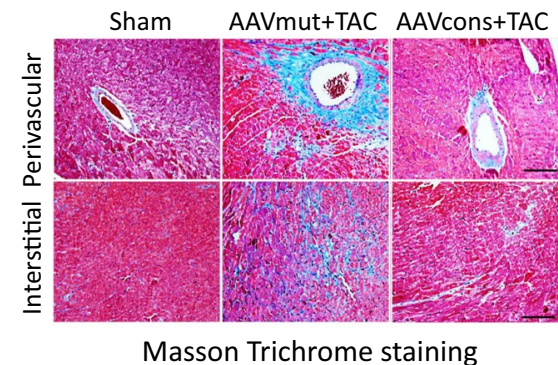

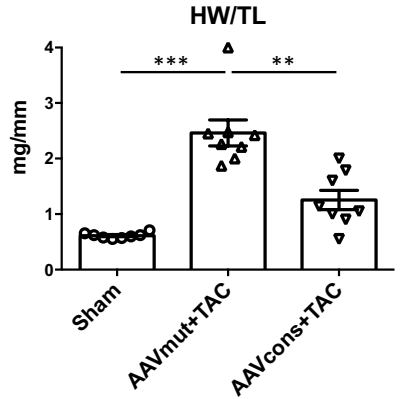

$f$

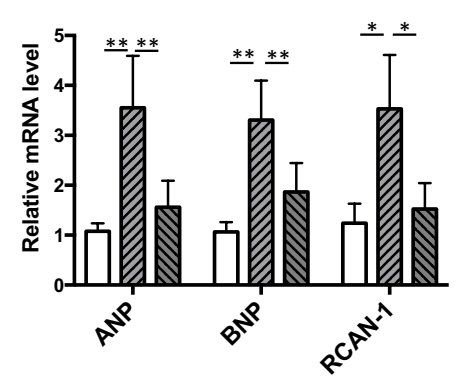

d

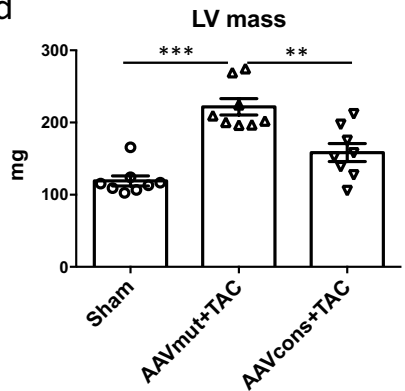

$\square$ Sham
$\square$ AAVmut+TAC g

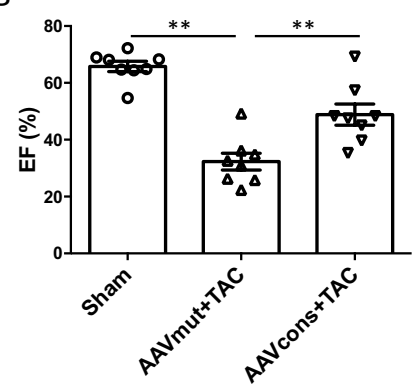

i j

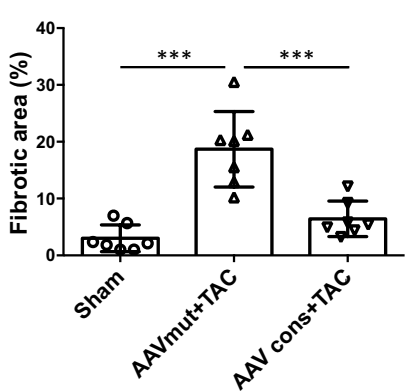

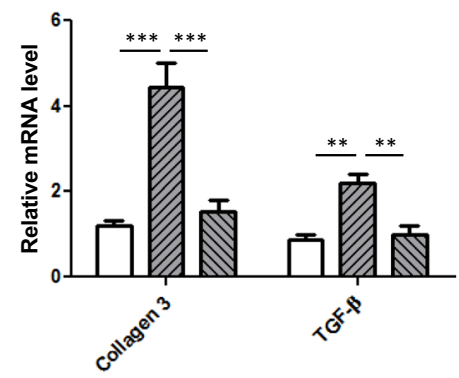

Fig. 3 AAV9-mediated generation of hpNFAT dONs prevent TAC-induced myocardial hypertrophy and dysfunction in mice. a Graphical representation of experimental timeline in the preventive approach. b Illustrative confocal images proving efficient hpNFAT dON production in cardiomyocytes after tail vein injection of AAV9. Scale bar represents $25 \mu \mathrm{m}$. c Statistical quantification of heart weight/tibia length (HW/TL) of mice subjected to the depicted treatments. d Measurement of left ventricular (LV) mass by echocardiography following TAC. e Statistical quantification of relative cardiomyocyte cross-sectional area, measured after WGA staining on cardiac frozen sections. Values were normalized to the sham group. f Gene expression analysis of hypertrophy markers ANP, BNP and RCAN-1 on the mRNA level by qPCR. RPL32 served as a housekeeping gene. g Myocardial function measured by ejection fraction 6 weeks after induction of cardiac hypertrophy. $\mathbf{h}$ Illustrative images showing Masson Trichrome staining of cardiac sections of the groups as indicated, and $\mathbf{i}$ statistical quantification of the percentage of blue area, marking pathological extracellular matrix deposition (20 images analyzed/ group, scale bar: $20 \mu \mathrm{m}$ ). j Relative expression levels of collagen 3 and TGF- $\beta$ as a further confirmation of fibrosis development after TAC $(n=8, * p<0.05, * * p<0.01, * * * p<0.001)$

animals was nearly abolished (Fig. 3f, Suppl. Figure 2c-e), and echocardiography revealed a significant improvement in cardiac function as well as a significant reduction in cardiac hypertrophy (Fig. 3g, Suppl. Figure 2f, g).

Fibrosis is a hallmark of heart failure and directly contributes to impairment of cardiac function by decreasing contractility and oxygenation [43]. AAV9-mediated expression of the mutant control ON prior to TAC resulted in a prominent increase both in perivascular and interstitial tion of the cardiac pro-hypertrophic gene program in these 
fibrosis as well as an increased expression of collagen 3 and transforming growth factor $\beta$ (TGF- $\beta$ ), which are prototypic for pressure overload-induced replacement fibrosis (Fig. 3h-j). All of these TAC-induced changes were essentially prevented following pre-treatment of the mice with the AAV9 expressing the consensus dON, further emphasizing its striking efficacy.

\section{AAV9-mediated cardiomyocyte-specific hpNFAT RNA decoy oligonucleotide expression abrogates TAC-induced cardiac hypertrophy and heart failure}

The cardioprotective effects of AAV9-mediated expression of the hpNFAT consensus dON were further analyzed in a therapeutic experimental setting. For this purpose, mice were injected with AAV9 vectors harboring the consensus or mutant control ON 3 days after TAC, as depicted in Fig. 4a. a

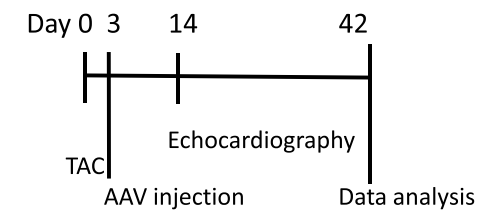

d

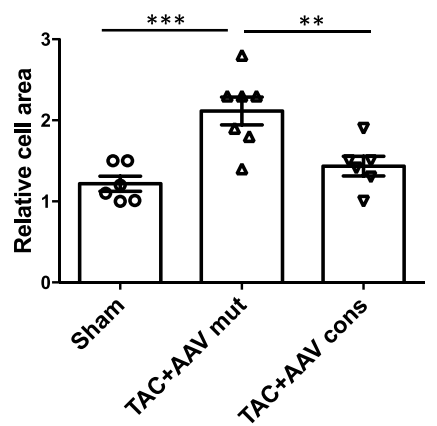

g

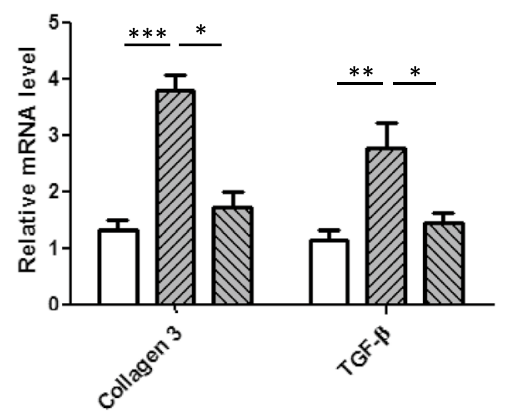

HW/TL

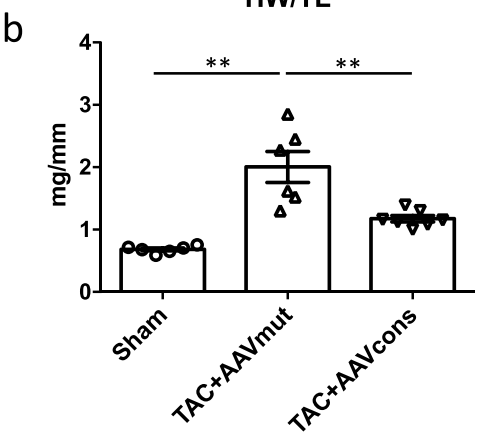

e

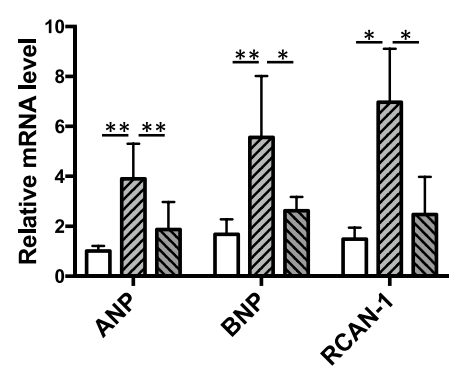

h

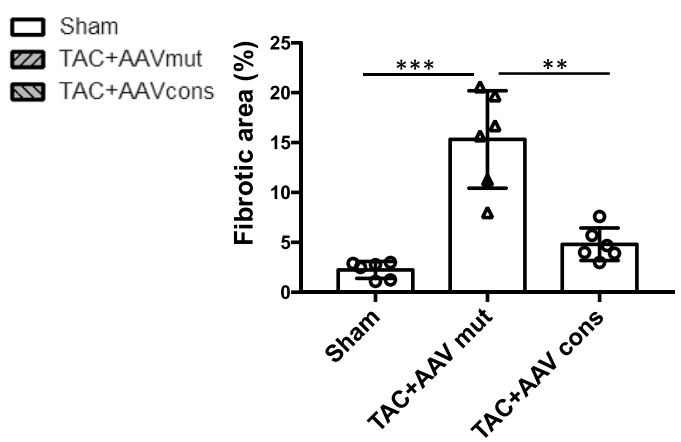

LV mass

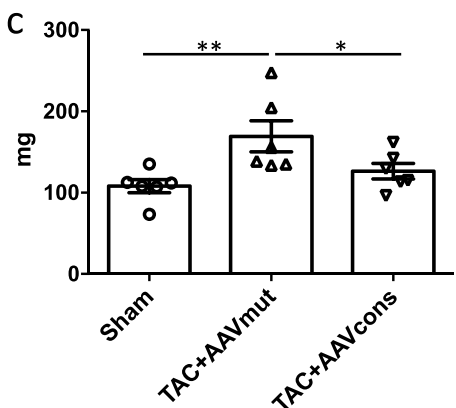

$f$

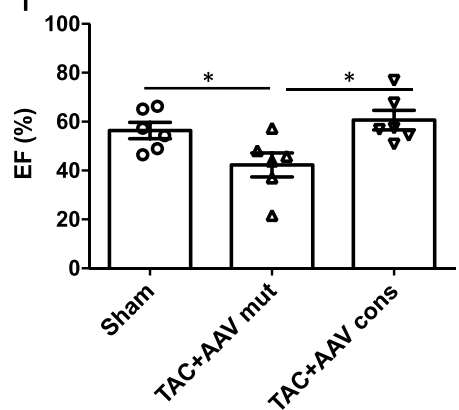

i

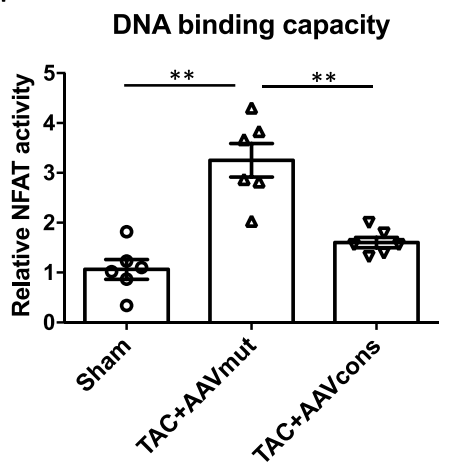

Fig. 4 AAV9-mediated delivery of hpNFAT dONs rescues cardiac dysfunction due to pressure overload in mice. a Timeline showing the experimental design of our in vivo experiment. b Quantification of heart weight/tibia length (HW/TL) ratio as a parameter of cardiac hypertrophy in the respective treatment groups. c Echocardiography-based measurement of left ventricular mass 6 weeks after TAC. d Statistical quantification of relative cell area of cardiac sections stained with WGA in the mentioned treatment groups. Values were normalized to the sham group. e Gene expression analysis of hypertrophic markers ANP, BNP and RCAN-1 in the myocardium of treated mice. RPL32 was used as a housekeeping gene and values were normalized to sham-operated mice. f Analysis of myocardial function measured by ejection fraction 6 weeks after TAC. g Statistical quantification of extracellular matrix deposition by Masson Trichrome staining of heart Sects. (20 images analyzed/group, scale bar: $20 \mu \mathrm{m})$. h Relative expression levels of collagen 3 and TGF- $\beta$ as markers of fibrosis. i Statistical quantification of NFAT activity following the specified treatments, 6 weeks after TAC $(n=6, * p<0.05$, $* * p<0.01, * * * p<0.001)$ 
As in the preventive experimental setting described above, TAC resulted in prominent cardiac hypertrophy, induction of the fetal gene program, systolic dysfunction, cardiac fibrosis and pro-fibrotic gene expression in the heart when the mice were treated with the mutant control ON expressing AAV9 3 days later (Fig. 4b-h, Suppl. Figure 3). In addition, there was a robust increase in nuclear NFAT activity in the cardiomyocytes 6 weeks after TAC (Fig. 4i, Suppl. Figure 4). Treatment with the consensus ON expressing AAV9 3 days post TAC essentially normalized all of the aforementioned parameters to the level in the sham-treated control animals, suggesting that AAV9-mediated expression of the hpNFAT $\mathrm{dON}$ is equally effective both in a preventive and therapeutic experimental setting.

Next, we aimed to investigate whether the described treatment affects NFAT target genes in non-target organs following AAV9 transduction. As expected for AAV9 vectors, we could detect EGFP expression primarily in the myocardium after systemic administration and to a lower extent also in skeletal muscle, liver, kidneys, and spleen (Suppl. Figure 5a). Interestingly, however, a decreased RCAN-1 mRNA level, indicative of the efficacy of the expressed $\mathrm{dON}$, was only detected in cardiac muscle, as presented in Suppl. Figure $5 \mathrm{~b}-\mathrm{d}$.

\section{Discussion}

Our study provides a novel and effective therapeutic option for cardiac hypertrophy and heart failure, consisting of AAVmediated delivery of a RNA-based dON neutralizing transcriptional activity of the NFATc1-c4 family of transcription factors. Following one-time systemic injection of the AAV9based viral vector, the decoy oligonucleotide is specifically expressed by cardiomyocytes as a shRNA bearing the consensus binding site of NFATc1-c4, which potently downregulates expression of NFAT-dependent pro-hypertrophic genes, hence preventing or inhibiting TAC-induced cardiac hypertrophy and subsequent heart failure. This novel therapeutic approach for delivery of dONs provides the opportunity to target specifically the myocardium, while avoiding non-specific adverse effects in other types of cells in the body.

Recent studies focusing on establishing the molecular mechanisms underlying cardiac hypertrophy have made it possible to advance new therapies and identify novel targets for gene therapy [6, 19]. Various transcription factors have emerged as critical players in the development/manifestation of this disease with various etiologies, making them attractive targets for gene therapy [5, 20, 35, 37, 44]. The NFAT family of transcription factors, except NFAT5, is an essential contributor to the progression of cardiac hypertrophy through governing changes in cardiomyocyte gene expression in response to a supra-physiological increase in wall stress (pressure overload) causing a maladaptive increase in cardiomyocyte size and rise in wall thickness ultimately leading to heart failure [36].

Several studies evidence the potential of DNA oligodeoxynucleotides as pharmacological tools to ameliorate symptoms of heart disease. Antisense ODNs directed against leukemia inhibitory factor (LIF) and cardiotrophin-1 were successful in downregulating angiotensin II-induced cardiac hypertrophy in vitro [40]. Moreover, a single systemic administration of antisense ODNs targeting phospholamban, a SERCA2a inhibitor, dramatically improved contractility in a pressure overload model of heart failure [34]. In addition, decreasing NF- $\mathrm{KB}$ target genes ameliorated cardiac remodeling associated with ischemia [23]. On the other hand, dODNs targeting transcriptional activity of GATA4 failed to ameliorate the endothelin-1 or phenylephrine-induced switch in cardiomyocyte phenotype in vitro, suggesting that disrupting the activity of this transcription factor is insufficient to observe a beneficial effect [38]. However, neutralization of GATA4 by administration of dODNs in vivo has not been validated. As the contribution of fibroblasts and vessels is increasingly acknowledged in cardiac hypertrophy, the role of distinct cardiac transcription factors, such as GATA4 or GATA6, needs to be considered also in non-cardiomyocytes [11]. Although NFAT plays a central role in cardiac hypertrophy, the approach described here may be tested on further transcription factor targets involved in induction of cardiac hypertrophy, such as the MEF2, HAND, SFR or MITF genes.

The pro-hypertrophic effects of NFAT family members c 1 to 44 have already been well established in cardiomyocytes. NFAT activity has been demonstrated to be substantially increased in patients with dilated cardiomyopathy [10] and in various animal models of pathological hypertrophy [28, 45]. Furthermore, decreasing NFAT activity through administration of an inhibitory peptide (VIVIT) was shown to be an effective treatment for pressure overload-induced cardiac hypertrophy in a rat animal model preventing increased heart weight and serum concentrations of brain natriuretic peptide (BNP) and atrial natriuretic peptide (ANP) [24]. Although this approach may be therapeutically relevant, repeated injections of the peptide would be required in patients due to its relatively short half-life. Moreover, high doses of the peptide had to be administered to attain the observed therapeutic effect, which does not exclude the risk for possible off-target effects in other organs. Drug-based approaches of NFAT inhibition may not only result in potential extracardiac side effects, but might also be limited by distinct effects on NFAT subtypes. For example, the pharmacological NFATc3 inhibitor A-285222 protected from endothelial dysfunction in a murine diabetes model [13] but does not block NFATc2 playing a central role in pathological myocardial hypertrophy [2]. 
A particular requirement for any transcription factor neutralizing agent is that it should be specifically directed to the target transcription factor. The sequences of the dODN and dON used herein were designed according to the consensus DNA-binding site of NFATc1-c4 [39] to specifically neutralize only these NFAT family members but not NFAT5, which binds to distinct DNA motifs [25, 39]. As shown herein, expression of NFAT5 target genes upregulated through exposure to osmotic stress was not affected by the anti-NFAT1-4 dON, emphasizing the target specificity of the chosen nucleic acid-based drug candidate.

AAV-mediated expression of anti-NFAT1-4 dONs allowed a long-term cardiac effect of the therapeutic nucleic acid compound following single vector administration in our study similar to subcutaneous injection of the VIVIT peptide [2]. The NFAT-inhibiting peptide VIVIT was successfully expressed using an AAV-based approach in a mouse model of Alzheimer's disease [18]. However, no vector-mediated expression of VIVIT has been investigated in experimental cardiac hypertrophy so far.

Although AAV9 vectors mediated a predominant cardiac gene expression, some extra-cardiac transduction was also detectable. Nevertheless, we could not detect evidence of downregulation of NFAT target genes outside the heart. A potential reason could be that the expressed dONs may be solely active when nuclear translocation of NFAT is initiated as in cardiac hypertrophy and heart failure. Since TAC does not result in NFAT activation in non-cardiac tissue as observed in inflammatory liver diseases and fibrosis [3] or skeletal muscle growth and development [4], the hpNFAT $\mathrm{dON}$ overexpression may have no extra-cardiac side effects at least in our model.

In addition to reduced cardiac hypertrophy and improved cardiac function, we noted an almost complete prevention or inhibition of cardiac fibrosis as well as pro-fibrotic gene expression in TAC mice with AAV9-mediated expression of the hpNFAT dON. This result is in line with previous studies showing that NFAT inhibition through injection of FTY720 , a compound used for treatment of multiple sclerosis, reverses cardiac fibrosis in mice subjected to TAC. Moreover, TGF- $\beta$-induced collagen 1 and fibronectin production was shown to be NFAT-dependent [48]. Interestingly, fibronectin itself was proven to induce NFAT activity [21].

Although the data provided here clearly suggest a promising and innovative treatment option for cardiac hypertrophy transiting to heart failure, we acknowledge that there may be considerable differences between small animal models for heart disease and the situation in humans. For this reason, testing the feasibility of this approach in a non-rodent mammalian system is required. This would potentially constitute a step forward in bringing the suggested gene therapy closer to clinical trials and patients.
Moreover, even though TAC is a well-characterized model for cardiac hypertrophy and heart failure, it is not without limitations and challenges with regard to translation into the human setting. This method causes a rapid induction of pressure overload, which might be different from gradual development of aortic stenosis in patients. In addition, mice subjected to TAC required rather early analysis to avoid loss due to increased mortality. Moreover, we have used young adult mice, which adapt faster to TAC than older animals [27]. In contrast, rat models of TAC present with a slower onset of left ventricular hypertrophy and disease progression [14]. Thus, as AAV vectors enable sustained cardiac gene expression, our approach should be studied regarding its long-term effects in additional models of cardiac disease involving abnormal activation of NFAT including pulmonary hypertension [7] or heart failure induced by myocardial infarction [45]. A further challenge for translation into the situation in patients is the requirement of choosing the right time point for vector application as repeated AAV application is limited by induction of neutralizing antibodies after the first injection.

In conclusion, our study is the first to show that a RNAbased dON targeting NFATc1-c4 can be generated in cardiomyocytes following AAV9 transduction, and can be employed both as a preventive as well as a therapeutic option for rescuing cardiac function in a pressure overload model in mice. Moreover, from a mechanistic point of view, our study undeniably unveils the importance of inhibiting cardiac hypertrophy to prevent transition into chronic heart failure.

Supplementary Information The online version contains supplementary material available at https://doi.org/10.1007/s00395-021-00880-w.

Acknowledgements We are grateful for the expert technical assistance and help provided by Franziska Mohr and Angela Schulz. This work was supported by grants from the Baden-Württemberg-Stiftung to M.H., the German Centre for Cardiovascular Research to N.F. and O.J.M. (81Z2700204, 81Z0700201) and the FAZIT foundation to A.R.

Authors' contributions OJM, HAK, NF, and MH initially conceived the project. AR performed most of the experiments under supervision of AHW, NF, MH and OJM. AHW conceived the decoy construct with input from AJ and OJM. AJ produced AAVs. FS and NU performed histological analyses. NS, M. Heckmann, TR, and LD carried out in vivo experiments, AR, NS, MH, AJ, FS, and NU analyzed data. $\mathrm{AR}, \mathrm{AHW}, \mathrm{MH}$ and OJM wrote the manuscript with input from all authors. All authors read and approved the final manuscript.

Funding Open Access funding enabled and organized by Projekt DEAL. This work was supported by grants from the Baden-Württemberg-Stiftung to M.H., the German Centre for Cardiovascular Research to N.F. and O.J.M. (81Z2700204, 81Z0700201) and the FAZIT foundation to A.R. 
Availability of data and material Any materials can be obtained from the authors upon request.

\section{Declarations}

Conflict of interest A.R., A.H.W., A.J., H.A.K., M.H., and O.J.M. have filed a patent application on the therapeutic use of hpNFAT dON treatment in patients with cardiac hypertrophy and heart failure.

Ethics approval Animal experiments were executed with approval of the local authorities in Kiel (permission number V312-7224.121-4 (756/14) and 1085) and Karlsruhe (G180/12).

Consent for publication All authors approved the final manuscript and agreed to publication in Basic Research in Cardiology.

Open Access This article is licensed under a Creative Commons Attribution 4.0 International License, which permits use, sharing, adaptation, distribution and reproduction in any medium or format, as long as you give appropriate credit to the original author(s) and the source, provide a link to the Creative Commons licence, and indicate if changes were made. The images or other third party material in this article are included in the article's Creative Commons licence, unless indicated otherwise in a credit line to the material. If material is not included in the article's Creative Commons licence and your intended use is not permitted by statutory regulation or exceeds the permitted use, you will need to obtain permission directly from the copyright holder. To view a copy of this licence, visit http://creativecommons.org/licenses/by/4.0/.

\section{References}

1. Bird L (2004) Lymphocyte responses - NFAT5: helping out in stressful times. Nat Rev Immunol 4:581-581. https://doi.org/10. 1038/nri1429

2. Bourajjaj M, Armand AS, da Costa Martins PA, Weijts B, van der Nagel R, Heeneman S, Wehrens XH, De Windt LJ (2008) NFATc2 is a necessary mediator of calcineurin-dependent cardiac hypertrophy and heart failure. J Biol Chem 283:22295-22303. https:// doi.org/10.1074/jbc.M801296200

3. Cai SY, Yu D, Soroka CJ, Wang J, Boyer JL (2021) Hepatic NFAT signaling regulates the expression of inflammatory cytokines in cholestasis. J Hepatol 74:550-559. https://doi.org/10.1016/j.jhep. 2020.09.035

4. Calabria E, Ciciliot S, Moretti I, Garcia M, Picard A, Dyar KA, Pallafacchina G, Tothova J, Schiaffino S, Murgia M (2009) NFAT isoforms control activity-dependent muscle fiber type specification. Proc Natl Acad Sci USA 106:13335-13340. https://doi.org/ 10.1073/pnas.0812911106

5. Camporeale A, Marino F, Papageorgiou A, Carai P, Fornero S, Fletcher S, Page BDG, Gunning P, Forni M, Chiarle R, Morello M, Jensen O, Levi R, Heymans S, Poli V (2013) STAT3 activity is necessary and sufficient for the development of immune-mediated myocarditis in mice and promotes progression to dilated cardiomyopathy. EMBO Mol Med 5:572-590

6. Cannata A, Ali H, Sinagra G, Giacca M (2020) Gene therapy for the heart lessons learned and future perspectives. Circ Res 126:1394-1414. https://doi.org/10.1161/CIRCRESAHA.120. 315855

7. Chen R, Yan J, Liu P, Wang Z, Wang C, Zhong W, Xu L (2017) The role of nuclear factor of activated $\mathrm{T}$ cells in pulmonary arterial hypertension. Cell Cycle 16:508-514. https://doi.org/10. 1080/15384101.2017.1281485

8. D'Acquisto F, Ialenti A, Ianaro A, Di Vaio R, Carnuccio R (2000) Local administration of transcription factor decoy oligonucleotides to nuclear factor- $\kappa \mathrm{B}$ prevents carrageenin-induced inflammation in rat hind paw. Gene Ther 7:1731-1737. https://doi.org/ 10.1038/sj.gt.3301295

9. Dajee M, Muchamuel T, Schryver B, Oo A, Alleman-Sposeto J, De Vry CG, Prasad S, Ruhrmund D, Shyamsundar R, Mutnick D, Mai K, Le T, Parham C, Zhang J, Komuves L, Colby T, Hudak S, McEvoy LM, Ehrhardt RO (2006) Blockade of experimental atopic dermatitis via topical NF- $\mathrm{KB}$ decoy oligonucleotide. J Invest Dermatol 126:1792-1803. https://doi.org/10.1038/sj.jid. 5700307

10. Diedrichs H, Chi M, Boelck B, Mehlhorn U, Schwinger RH (2004) Increased regulatory activity of the calcineurin/NFAT pathway in human heart failure. Eur J Heart Fail 6:3-9. https:// doi.org/10.1016/j.ejheart.2003.07.007

11. Dittrich GM, Froese N, Wang X, Kroeger H, Wang H, Szaroszyk M, Malek-Mohammadi M, Cordero J, Keles M, Korf-Klingebiel M, Wollert KC, Geffers R, Mayr M, Conway SJ, Dobreva G, Bauersachs J, Heineke J (2021) Fibroblast GATA-4 and GATA-6 promote myocardial adaptation to pressure overload by enhancing cardiac angiogenesis. Basic Res Cardiol 116:26. https://doi.org/ 10.1007/s00395-021-00862-y

12. Frey N, Olson EN (2003) Cardiac hypertrophy: the good, the bad, and the ugly. Annu Rev Physiol 65:45-79. https://doi.org/10.1146/ annurev.physiol.65.092101.142243

13. Garcia-Vaz E, McNeilly AD, Berglund LM, Ahmad A, Gallagher JR, Dutius Andersson AM, McCrimmon RJ, Zetterqvist AV, Gomez MF, Khan F (2020) Inhibition of NFAT signaling restores microvascular endothelial function in diabetic mice. Diabetes 69:424-435. https://doi.org/10.2337/db18-0870

14. Gs AK, Raj B, Santhosh KS, Sanjay G, Kartha CC (2014) Ascending aortic constriction in rats for creation of pressure overload cardiac hypertrophy model. J Vis Exp. https://doi.org/10.3791/ 50983

15. Hecker M, Wagner AH (2017) Transcription factor decoy technology: a therapeutic update. Biochem Pharmacol 144:29-34. https:// doi.org/10.1016/j.bcp.2017.06.122

16. Heckmann MB, Bauer R, Jungmann A, Winter L, Rapti K, Strucksberg KH, Clemen CS, Li Z, Schroder R, Katus HA, Muller OJ (2016) AAV9-mediated gene transfer of desmin ameliorates cardiomyopathy in desmin-deficient mice. Gene Ther 23:673-679. https://doi.org/10.1038/gt.2016.40

17. Hogan PG, Chen L, Nardone J, Rao A (2003) Transcriptional regulation by calcium, calcineurin, and NFAT. Genes Dev 17:22052232. https://doi.org/10.1101/gad.1102703

18. Hudry E, Wu HY, Arbel-Ornath M, Hashimoto T, Matsouaka R, Fan Z, Spires-Jones TL, Betensky RA, Bacskai BJ, Hyman BT (2012) Inhibition of the NFAT pathway alleviates amyloid beta neurotoxicity in a mouse model of Alzheimer's disease. J Neurosci 32:3176-3192. https://doi.org/10.1523/JNEUROSCI.6439-11. 2012

19. Kaufmann KB, Buning H, Galy A, Schambach A, Grez M (2013) Gene therapy on the move. EMBO Mol Med 5:1642-1661. https:// doi.org/10.1002/emmm.201202287

20. Kohli S, Ahuja S, Rani V (2011) Transcription factors in heart: promising therapeutic targets in cardiac hypertrophy. Curr Cardiol Rev 7:262-271

21. Konstandin MH, Volkers M, Collins B, Quijada P, Quintana M, De La Torre A, Ormachea L, Din S, Gude N, Toko H, Sussman MA (2013) Fibronectin contributes to pathological cardiac hypertrophy but not physiological growth. Basic Res Cardiol 108:375. https://doi.org/10.1007/s00395-013-0375-8 
22. Kucera JP, Prudat Y, Marcu IC, Azzarito M, Ullrich ND (2015) Slow conduction in mixed cultured strands of primary ventricular cells and stem cell-derived cardiomyocytes. Front Cell Dev Biol 3:58. https://doi.org/10.3389/fcell.2015.00058

23. Kupatt C, Wichels R, Deiss M, Molnar A, Lebherz C, Raake P, von Degenfeld G, Hahnel D, Boekstegers P (2002) Retroinfusion of NFKB decoy oligonucleotide extends cardioprotection achieved by CD18 inhibition in a preclinical study of myocardial ischemia and retroinfusion in pigs. Gene Ther 9:518-526. https://doi.org/ $10.1038 /$ sj.gt.3301673

24. Kuriyama M, Matsushita M, Tateishi A, Moriwaki A, Tomizawa K, Ishino K, Sano S, Matsui H (2006) A cell-permeable NFAT inhibitor peptide prevents pressure-overload cardiac hypertrophy. Chem Biol Drug Des 67:238-243. https://doi.org/10.1111/j.17470285.2006.00360.x

25. Lee N, Kim D, Kim WU (2019) Role of NFAT5 in the immune system and pathogenesis of autoimmune diseases. Front Immunol 10:270. https://doi.org/10.3389/fimmu.2019.00270

26. Lehmann LH, Rostosky JS, Buss SJ, Kreusser MM, Krebs J, Mier W, Enseleit F, Spiger K, Hardt SE, Wieland T, Haass M, Luscher TF, Schneider MD, Parlato R, Grone HJ, Haberkorn U, Yanagisawa M, Katus HA, Backs J (2014) Essential role of sympathetic endothelin A receptors for adverse cardiac remodeling. Proc Natl Acad Sci USA 111:13499-13504. https://doi.org/10.1073/pnas. 1409026111

27. Li YH, Reddy AK, Ochoa LN, Pham TT, Hartley CJ, Michael LH, Entman ML, Taffet GE (2003) Effect of age on peripheral vascular response to transverse aortic banding in mice. J Gerontol A Biol Sci Med Sci 58:B895-899. https://doi.org/10.1093/gerona/58.10. b895

28. Liu Q, Chen Y, Auger-Messier M, Molkentin JD (2012) Interaction between NFkappaB and NFAT coordinates cardiac hypertrophy and pathological remodeling. Circ Res 110:1077-1086. https://doi.org/10.1161/CIRCRESAHA.111.260729

29. Mann MJ, Dzau VJ (2000) Therapeutic applications of transcription factor decoy oligonucleotides. J Clin Invest 106:1071-1075. https://doi.org/10.1172/JCI11459

30. Metra M, Teerlink JR (2017) Heart failure. Lancet 390:19811995. https://doi.org/10.1016/S0140-6736(17)31071-1

31. Mognol GP, Gonzalez-Avalos E, Ghosh S, Spreafico R, Gudlur A, Rao A, Damoiseaux R, Hogan PG (2019) Targeting the NFAT:AP-1 transcriptional complex on DNA with a small-molecule inhibitor. Proc Natl Acad Sci USA 116:9959-9968. https:// doi.org/10.1073/pnas.1820604116

32. Molkentin JD (2004) Calcineurin-NFAT signaling regulates the cardiac hypertrophic response in coordination with the MAPKs. Cardiovasc Res 63:467-475. https://doi.org/10.1016/j.cardiores. 2004.01.021

33. Molkentin JD, Lu JR, Antos CL, Markham B, Richardson J, Robbins J, Grant SR, Olson EN (1998) A calcineurin-dependent transcriptional pathway for cardiac hypertrophy. Cell 93:215-228

34. Morihara $\mathrm{H}$, Yamamoto T, Oiwa H, Tonegawa K, Tsuchiyama D, Kawakatsu I, Obana M, Maeda M, Mohri T, Obika S, Fujio Y, Nakayama H (2017) Phospholamban inhibition by a single dose of locked nucleic acid antisense oligonucleotide improves cardiac contractility in pressure overload-induced systolic dysfunction in mice. J Cardiovasc Pharmacol Ther 22:273-282. https://doi.org/ $10.1177 / 1074248416676392$
35. Morin S, Charron F, Robitaille L, Nemer M (2000) GATAdependent recruitment of MEF2 proteins to target promoters. EMBO J 19:2046-2055. https://doi.org/10.1093/emboj/19.9.2046

36. Nakamura M, Sadoshima J (2018) Mechanisms of physiological and pathological cardiac hypertrophy. Nat Rev Cardiol 15:387407. https://doi.org/10.1038/s41569-018-0007-y

37. O'Sullivan KE, Breen EP, Gallagher HC, Buggy DJ, Hurley JP (2016) Understanding STAT3 signaling in cardiac ischemia. Basic Res Cardiol 111:27. https://doi.org/10.1007/s00395-016-0543-8

38. Pikkarainen S, Kerkela R, Pontinen J, Majalahti-Palviainen T, Tokola H, Eskelinen S, Vuolteenaho O, Ruskoaho H (2002) Decoy oligonucleotide characterization of GATA-4 transcription factor in hypertrophic agonist induced responses of cardiac myocytes. J Mol Med (Berl) 80:51-60. https://doi.org/10.1007/s001090100 278

39. Rao A, Luo C, Hogan PG (1997) Transcription factors of the NFAT family: regulation and function. Annu Rev Immunol 15:707-747. https://doi.org/10.1146/annurev.immunol.15.1.707

40. Sano M, Fukuda K, Kodama H, Pan J, Saito M, Matsuzaki J, Takahashi T, Makino S, Kato T, Ogawa S (2000) Interleukin-6 family of cytokines mediate angiotensin II-induced cardiac hypertrophy in rodent cardiomyocytes. J Biol Chem 275:29717-29723. https:// doi.org/10.1074/jbc.M003128200

41. Schott P, Asif AR, Graf C, Toischer K, Hasenfuss G, Kogler H (2008) Myocardial adaptation of energy metabolism to elevated preload depends on calcineurin activity : a proteomic approach. Basic Res Cardiol 103:232-243. https://doi.org/10.1007/ s00395-008-0696-1

42. Shimizu I, Minamino T (2016) Physiological and pathological cardiac hypertrophy. J Mol Cell Cardiol 97:245-262. https://doi. org/10.1016/j.yjmcc.2016.06.001

43. Travers JG, Kamal FA, Robbins J, Yutzey KE, Blaxall BC (2016) Cardiac fibrosis: the fibroblast awakens. Circ Res 118:1021-1040. https://doi.org/10.1161/CIRCRESAHA.115.306565

44. Valen $G$ (2004) Signal transduction through nuclear factor $\kappa B$ in ischemia-reperfusion and heart failure. Basic Res Cardiol 99:1-7. https://doi.org/10.1007/s00395-003-0442-7

45. Wilkins BJ, Dai YS, Bueno OF, Parsons SA, Xu J, Plank DM, Jones F, Kimball TR, Molkentin JD (2004) Calcineurin/NFAT coupling participates in pathological, but not physiological, cardiac hypertrophy. Circ Res 94:110-118. https://doi.org/10.1161/ 01.RES.0000109415.17511.18

46. Wilkins BJ, De Windt LJ, Bueno OF, Braz JC, Glascock BJ, Kimball TF, Molkentin JD (2002) Targeted disruption of NFATc3, but not NFATc4, reveals an intrinsic defect in calcineurin-mediated cardiac hypertrophic growth. Mol Cell Biol 22:7603-7613. https://doi.org/10.1128/mcb.22.21.7603-7613.2002

47. Zhang QJ, Goddard M, Shanahan C, Shapiro L, Bennett M (2002) Differential gene expression in vascular smooth muscle cells in primary atherosclerosis and in stent stenosis in humans. Arterioscler Thromb Vasc Biol 22:2030-2036

48. Zimmerman KA, Graham LV, Pallero MA, Murphy-Ullrich JE (2013) Calreticulin regulates transforming growth factor-betastimulated extracellular matrix production. J Biol Chem 288:14584-14598. https://doi.org/10.1074/jbc.M112.447243 